\title{
Treatment of Depersonalization-Derealization Disorder With Transcranial Magnetic Stimulation
}

Cândida Coelho, , Gonçalo Santos ${ }^{1,2}$, Sara Magano $0^{1,2}$, Joana Andrade ${ }^{1,2}$, David Motal,2, Sofia Ferreiral,2, Ana Araújo $0^{1,2}$, Carolina Cabaços ${ }^{1,2}$, António Macedo ${ }^{1,2}$

1 University of Coimbra, Faculty of Medicine, ${ }^{2}$ Centro Hospitalar e Universitário de Coimbra (CHUC), Department of Psychiatry

\section{INTRODLCTION}

Depersonalization-Derealization Disorder (DDD) envolves recurrent or persistent experiences of unreality, emotional numbness and alterations in the sense of self. It often begins in adolescence and tends to chronify. Symptoms of depersonalization or derealization may also be transiente phenomenons in other psychiatric disorders. Over the time various drugs have been tested as possible therapeutic approaches in DDD. The inconsistent results and limited benefit led to the search for alternative therapies.

\section{OBJECTIVES}

To review the literature on the latest data related to Transcranial Magnetic Stimulation (TMS) in the treatment of DDD.

\section{MATERIALS ANID METHOIDS}

We present a classical review of the literature, based on a selection of scientific articles published in english between 1999 and 2019, collected in the databases PubMed, The Cochrane Library, as well as in relevant scientific journals in this area of research. In this work were used the following MeSH terms: "Depersonalization Disorder"; "Derealization"; "Transcranial Magnetic Stimulation".

\section{RESULTS}

Several clinical trials and case reports have shown promising results in the stimulation of prefrontal or temporoparietal junction using TMS in patients with DDD. Most of the papers used the reduction in Cambridge Depersonalization Scale (CDS) as the main outcome.

\section{CASE REPORTS}

Patients with Depersonalization-Derealization Disorder

- Male patient with 26 of age, diagnosed with DDD with 6 moths of evolution who responded with TMS in the right temporoparietal junction (TPJ) (reduction in CDS $>50 \%)^{\prime}$.

\section{Patients with DDD comorbid with Major Depressive Disorder}

- Male patient with 30 years of age who did not respond to pharmacotherapy was treated with rTMS to the left dorsolateral prefrontal cortex (DLPFC) with significant reduction of depersonalization symptoms (reduction in CDS $>50 \%)^{2}$.

- Male patient, 25 years-old, who underwent six sessions on the DLPFC, with a $28 \%$ reduction on $\mathrm{CDS}^{3}$.

\section{CLINICAL TRIALS}

- 7 patients submitted to one session of TMS on the right ventrolateral prefrontal cortex (VLPFC) , 2 were classified as "full responders" (reduction in CDS $>50 \%$ ), 4 as "partial responders" (reduction in CDS 25-50\%) and I as "non responder" (reduction in CDS $<25 \%){ }^{4}$

- 6 patients with DDD submitted to TMS in the right TPJ with 3 weeks duration with positive response ${ }^{5}$.

- 17 patients with DDD resistant to therapeutic have shown symptomatic reduction after one session of TMS in the right VLPFC or right TPJ ${ }^{6}$.

- 12 patients with DDD resistant to therapeutic have shown symptomatic redution after repetitive TMS in the right TJP; 6 were classified as responders (4 "full responders" and 2 "partial responders")2.

\section{DISCLSSION}

Several clinical trials and case reports have shown promising results in the use of TMS over the prefrontal and temporoparietal regions in patients with DDD.

Most of the clinical trials performed were single center and included individuals with Primary Dissociative Disorder, all of them with demonstrated significant improvement of the described symptoms. A number of successful cases have also been described in individuals with depressive disorders and comorbid DDD, also with significant clinical improvement. To date there are no randomized controlled trials or multicenter studies performed.

Further studies will also clarify the already developed relationship between the neurobiology of the disease and the areas commonly used as therapeutic targets. It has been proposed that the sense of emotional numbing reported by patients may be due to a fronto-limbic malfunctioning (namely, an abnormally increased frontal inhibitory regulation on emotion-generation areas as the limbic structures, affecting the response to emotional stimuli). In turn, feelings of disembodiment and lack of agency may be related with temporoparietal dysfunction, a multilmodal region with a crucial role in generating a "sense of self" and in self-non-self distinction.

\section{CONCLISIONS}

Several studies suggest promising results in the use of TMS in patients with DDD. However, randomized and controlled trials will be necessary in order to validate this data and allow its use in clinical practice.

\section{REFERENCES}

I. Rachid F. Treatment of a Patient With Depersonalization Disorder With Low Frequency Repetitive Transcranial Magnetic Stimulation of the Right Temporo-Parietal Junction in a Private Practice Setting. Journal of Psychiatric Practice 2017;23;145147

2. Karris BC et al. Treatment of Depersonalization Disorder With Repetitive Iranscranial Magnetic Stimulation. Journal of Psychiatric Practice 2017;23;14I- 144

3. Jimenez-Genchi AM. Repetitive Iranscranial Magnetic Stimulation Improves Depersonalization: A Case Report. CNS Spectr. 2004;9(5)-375-376

4. Jay EL et al. Ventrolateral prefrontal cortex repetitive transcranial magnetic stimulation in the treatment of depersonalization disorder: A consecutive case series. Psychiatry Research. 2016.240. (II8-122)

5. Mantovani A, et al. Temporo-parietal junction stimulation in the treatment of depersonalization disorder. Psychiatry Research 186 (2011) $138-140$

6. Jay EL et al. Testing a neurobiological model of depersonalization disorder using repetitive transcranial magnetic stimulation. Brain Stimulation 7 (2014) 2522259

7. Berman J. Commentary. Iranscranial Magnetic Stimulation: Does It Matter Where or What or Whom You Target? Journal of Psychiatric Practice 2017;23;148- 149

8. Donaldson P, et al. Noninvasive stimulation of the temporoparietal junction: A systematic review. Neurosci. Biobehav. Rev. (2015) 